\title{
Is cognitive control automatic? New insights from transcranial magnetic stimulation
}

\author{
G. Cona ${ }^{1} \cdot$ B. Treccani ${ }^{2} \cdot$ C. A. Umiltà ${ }^{1}$
}

Published online: 11 March 2016

(C) Psychonomic Society, Inc. 2016

\begin{abstract}
Cognitive control has been classically considered as a flexible process engaged to pursue intentional behaviors, as distinct from automatic processes, which are unintentional, inflexible, and triggered by unconscious mechanisms. Our study challenged this view, showing that such a distinction may not be so clear-cut. We analyzed motor-evoked potentials (MEPs) elicited by transcranial magnetic stimulation to investigate the neurocognitive mechanisms occurring in a conflict task during trials that either required or did not require a response. We observed a Simon effect on MEPs and sequential modulations of such effects on both kinds of trials. Sequential modulations are usually explained as resulting from the engagement of intentional control mechanisms. Our findings rule against this idea, suggesting that these effects are the result of a mechanism that detects and resolves conflict even when there is no intention to select any response. Accordingly, cognitive control also seems to operate without intention, acting in an automatic fashion.
\end{abstract}

$\overline{\text { G. Cona and B. Treccani contributed equally and should be considered }}$ co-first-authors.

\section{G. Cona \\ giorgia.cona@unipd.it \\ B. Treccani \\ btreccani@uniss.it \\ C. A. Umiltà \\ carlo.umilta@unipd.it}

1 Department of General Psychology, University of Padua, Via Venezia, 8, 35131 Padova, Italy

2 Department of History, Human Sciences and Education, University of Sassari, Via Maurizio Zanfarino, 62, 07100 Sassari, Italy
Keywords Cognitive control $\cdot$ Automatic $\cdot$ TMS $\cdot$ Conflict task $\cdot$ MEPs $\cdot$ Simon effect $\cdot$ Sequential modulations · Intentionality

In everyday life, we encounter several conflicting situations. To ensure successful task performance, we need to detect and resolve conflict in information processing via a control system, which modulates cognitive processes accordingly. In the context of response selection, cognitive control is thought to be engaged when a response has to be selected from among competing alternatives to accomplish a consciously, internally activated goal; thus, it is typically considered to act only when intentional goal-directed actions are required (Botvinick, Braver, Barch, Carter, \& Cohen, 2001; Egner \& Hirsch, 2005). Yet, it is still not very clear what levels of consciousness and intentionality (cf. Bargh, 1990) are required for the cognitive control mechanism to be activated.

A hallmark of the paradigms used to explore cognitive control is that distinct responses can be simultaneously activated by the task-relevant and -irrelevant attributes of the stimulus, giving rise to a conflict (Lu \& Proctor, 1995). On compatible trials, the same response is activated by both the task-irrelevant attribute, via a direct, automatic route, and the task-relevant attribute, via an indirect controlled route. On incompatible trials, the two processing routes activate different responses, leading to a conflict. For example, in Simon tasks, participants have to respond to a nonspatial attribute of the stimulus (e.g., its color), which can appear in different positions. Even if the stimulus position is irrelevant to the task, responses are faster and more accurate when the response and stimulus positions correspond (i.e., compatible S-R trials; e.g., the stimulus appears on the right and a right key press is required) than when they do not (i.e., incompatible S-R trials; e.g., the stimulus appears on the left and a right key press is required). Another 
robust finding is that this effect (the so-called Simon effect) is reduced, absent, or even reversed when the preceding trial is incompatible (Ridderinkhof, 2002).

The sequential modulation of the Simon effect is typically explained as the result of the engagement of a control mechanism that acts to monitor and solve response conflict (i.e., the conflict adaptation account; Akçay \& Hazeltine, 2007). Following this view, a useful way to investigate whether the activation of a control mechanism implies awareness of conflict and an intention to resolve it consists in examining the conditions under which such sequential effects emerge.

Previous studies have already shown that conflict awareness is not critical to activate cognitive control. Notconsciously-perceived stimuli can activate a response that conflicts with the correct one, and the resulting (unconscious) conflict has been shown to trigger cognitive control mechanisms ${ }^{1}$ (Desender \& Van Den Bussche, 2012; Eimer \& Schlaghecken, 1998; Lau \& Passingham, 2007; Mayr, 2004; van Gaal, Lamme, \& Ridderinkhof, 2010). In all of these studies, however, the tasks required participants to emit a certain response. Conversely, it is still unclear what happens when no response needs to be selected and performed - thus, when the resolution of a possible conflict, either consciously or unconsciously generated, is not required. Just as it has been shown that cognitive control can be activated in the absence of awareness of the conflict, one might conjecture that it might be activated even if it is not necessary, and thus when there is no intention to act.

According to the conflict adaptation account, during the execution of conflict tasks, such as the Simon task, a control mechanism is activated to allow us to select the response we intend to execute. In incompatible trials, it would detect response conflict and suppress the direct route, which is responsible for the activation of the incorrect response. The suppression of the direct route would lead, in turn, to a reduction of the Simon effect in the following trial (e.g., Ansorge, Fuchs, Khalid, \& Kunde, 2011). In line with this view, sequential modulations of the Simon effect should occur only when we have to respond and want to avoid the selection of the incorrect response, whereas it should not be observed when no response has to be selected.

To test this hypothesis, we made use of a Simon task in which trials were organized in pairs of two consecutive responding or nonresponding trials, and we applied singlepulse transcranial magnetic stimulation (TMS) in the second trial of each pair. This technique allowed us to investigate

\footnotetext{
${ }^{1}$ Sequential effects observed when irrelevant stimuli in $N-1$ trials are not consciously detected have usually been interpreted as suggesting that unconsciously generated response conflicts can trigger cognitive control mechanisms. However, there are alternative interpretations of these findings. For example, it has been proposed that these effects result from an adaptation of response initiation processes in the current trial to the difficulty of the previous trial (see, e.g., Kinoshita, Mozer, \& Forster, 2011).
}

response tendencies even in trials that did not require a response and that followed a trial $N-1$ in which neither a response was given nor TMS used.

TMS over the primary motor cortex (M1) has been used to study the neurocognitive dynamics underlying conflict monitoring and cognitive control (Klein, Petitjean, Olivier, \& Duque, 2014; Michelet, Duncan, \& Cisek, 2010) and is the elective tool used to elucidate covert aspects of motor activity (Ticini, Schütz-Bosbach, Weiss, Casile, \& Waszak, 2012). When applied over M1, at appropriate intensities, the TMS pulse brings motor cortical neurons over the threshold. The descending volley reaches the spinal motor neurons, eliciting a motor-evoked potential (MEP). The amplitude of TMSevoked MEPs can be used as a measure of cortical excitability. The activation of a response (even when it is not followed by the emission of an actual response) is indexed by an increase in the excitability of the contralateral M1, which leads to an increase in the likelihood of eliciting a MEP and/or in the size of the MEP elicited. In choice reaction time (RT) tasks, for example, stimulation of the area of M1 controlling an activated response leads to larger MEPs than with a nonactivated response (Michelet et al., 2010). Recent TMS studies have revealed the dynamic pattern of M1 excitability underlying the Simon effect and its sequential modulations. These studies showed that MEPs recorded over the hand ipsilateral to the stimulus position were larger than those recorded over the contralateral hand (Stürmer, Siggelkow, Dengler, \& Leuthold, 2000), and that this difference in MEP amplitudes (i.e., a Simon effect) was modulated by the spatial compatibility in the preceding trial $N-1$, with it being greatest after compatible trials, intermediate after neutral trials, and smallest after incompatible trials (Treccani, Cona, Milanese, \& Umiltà, 2016; van Campen, Keuken, van den Wildenberg, \& Ridderinkhof, 2014).

In the present study, we recorded TMS-evoked MEPs to evaluate the possible changes of M1 excitability in the second trials of both responding and nonresponding pairs. In nonresponding pairs, participants, albeit being presented with lateralized stimuli, did not have to emit any response. This paradigm allowed us to investigate whether the direct and controlled routes were also activated in this kind of trial, and if so, whether a conflict occurred between the responses activated by the two routes, in spite of the fact that there was no need to select any response. Finally, we verified whether cognitive control could have been unintentionally engaged to resolve such conflict. An investigation of the Simon effect on MEP amplitudes and its sequential modulation allowed us to clarify these questions.

Within the context of the conflict adaptation account, several predictions can be made. A possibility might be that, in nonresponding trials, the direct route, being automatic, is activated, whereas the controlled route is not, given that no overt response is required. This should result in increased activation only of the M1 controlling the hand ipsilateral to the stimulus 
location. Alternatively, the controlled route might become activated, as well. The activation of both the direct and controlled routes should lead to a response conflict on incompatible trials. Obviously, there would be no need to resolve such conflict. Nevertheless, if the conflict were somehow detected and triggered an automatic control mechanism aimed at resolving it, we should observe a sequential modulation of the compatibility effects on MEPs. By contrast, we did not expect to find any sequential modulation of compatibility effects if the detection and resolution of response conflict can only be mediated by a mechanism tightly related to the willingness to resolve it in order to select the correct response.

\section{Method}

\section{Participants}

Eighteen students (11 females, seven males) at the University of Padua, with a mean age of 26 years (range: 21-29 years), took part in this study. There were 15 right-handers and three left-handers according to the Edinburgh Handedness Inventory (Oldfield, 1971). The participants had normal or corrected-to-normal vision and no neurological history or psychiatric disorder. They were checked for TMS exclusion criteria (Wassermann, 1998) and gave their informed written consent. The study was conducted in agreement with the Declaration of Helsinki and was approved by the local ethics committee.

\section{Task}

Participants were seated in front of a color monitor screen. The stimulus was a green or red square presented on a black background to the left or right of the fixation point, or was aligned vertically with fixation either above (for half of the participants) or below (for the other half) the fixation. At the beginning of the experiment, participants were given the instructions. They were informed that the task was composed of pairs of trials and that they had to respond by pressing one of two possible keys (one on the left and one on the right) with the left or right index finger. On half of the pairs (i.e., responding trials), they were required to respond to the red square by pressing either the left or the right key (the stimulus colorresponse mapping was counterbalanced across participants) and to the green square by pressing the other key. Trials wherein the stimulus occurred on the left or the right could be either spatially compatible or incompatible trials, whereas the trials wherein the stimulus was centrally presented were the neutral trials. For the other half of the pairs (i.e., nonresponding trials), participants were required to simply look at the squares, keeping their hands relaxed without performing any response. Nonresponding trials were considered to be spatially compatible, incompatible, or neutral as a function of the stimulus-response mapping that was valid in the responding trials. Before each pair of trials, instructions appeared on the screen and indicated to the participants whether to respond to both of the two subsequent stimuli (rispondi), or not to perform any response in either of the two subsequent trials $(x x x x)$. It is important to underline that nonresponding trials were not "no-go" trials; indeed, no-go trials are trials on which participants are ready to respond but the stimulus value on a certain dimension signals that no response has to be emitted. Conversely, in the nonresponding trials of our task, the stimulus-relevant values were associated with specific responses, but participants were informed well in advance not to respond (the instructions remained on the screen for $1,800 \mathrm{~ms}$ before the onset of the first stimulus of the pair). Indeed, no commission error was ever observed across participants. A practice run was administered at the beginning of the experiment.

\section{TMS protocol}

TMS was delivered through a figure $870-\mathrm{mm}$ coil and a Magstim 200 stimulator. A single TMS pulse was applied over left and right M1 with an intensity of $100 \%$ of the individual threshold at rest. This threshold was defined as the minimum intensity that induced five MEPs out of ten successive stimulation trials at rest (Rossini et al., 1994). The resulting mean intensity was $52.22 \%$ (range: $45 \%-67 \%$ ) of the maximum stimulator output.

TMS was applied $200 \mathrm{~ms}$ after the onset of the stimulus, on the basis of lateralized readiness potential findings revealing greater activation of the area of M1 controlling the hand ipsilateral to the stimulus position at this time point (Valle-Inclán, 1996).

The experiment was composed of two sessions, in which TMS was delivered separately to left and right M1. The order of stimulation was counterbalanced between participants. Each experimental session comprised 216 pairs of trials, divided in four blocks. TMS was delivered on $66.6 \%$ of the pairs of trials and was applied on the second trial of each pair. Thus, behavioral and MEP measures were analyzed only for the second trials of the pairs.

\section{MEP recording and analysis}

Electromyographic signal was recorded using cup electrodes positioned on the left and right hands in a bipolar montage, with the active electrode being placed over the first dorsal interosseus muscle and the inactive electrode over the second phalanx of the index of the same hand. The signal was amplified and digitized (sampling rate: $2048 \mathrm{~Hz}$ ). The electrode impedance was kept below $10 \mathrm{k} \Omega$, and the ground was placed on the arm. 
Only trials with TMS were included in the analysis. The signal was filtered (bandpass filter: 5-600 Hz) and epoched using $200 \mathrm{~ms}$ pre-TMS-pulse as the baseline. Amplitudes of the MEPs in responding and nonresponding trials were calculated and averaged separately for each experimental condition. The difference between the maximum and minimum peak values was recorded in the time window between 5 and $50 \mathrm{~ms}$ after the TMS pulse. In line with a large number of studies that analyzed MEP amplitudes (e.g., Cheeran et al., 2008; De Gennaro et al., 2007; Iyer, Schleper, \& Wassermann, 2003; Wassermann, 2002), these values were transformed into natural logarithms (Nielsen, 1996) to best fit the analysis of variance (ANOVA) assumptions.

\section{Results}

The RTs and accuracy on responding trials were analyzed by two ANOVAs, which included Spatial Compatibility in the Current $(N)$ Trial (compatible vs. incompatible) and Spatial Compatibility in the Preceding $(N-1)$ Trial (compatible, incompatible, and neutral) as within-subjects factors.

Both ANOVAs showed a significant main effect of the spatial compatibility in trial $N$ and a significant interaction between the two factors, revealing the presence of sequential modulations of the Simon effect in the behavioral data (see Table 1). Post-hoc comparisons (Fisher's least significant difference) showed that the Simon effect (i.e., the difference between RTs and proportions of correct responses in compatible and incompatible trials) was significant after compatible trials $(p<.05)$, reduced but still significant after neutral trials $(p<.05)$, and no longer significant after incompatible trials $(p>.05)$.

Amplitudes of the MEPs elicited in both responding and nonresponding trials were entered into an ANOVA with five within-participants factors: Type of Trial (responding vs. nonresponding trial), Stimulated Hemisphere (left vs. right), Hand Correctness (i.e., whether, in both responding and nonresponding trials, the hand from which the MEPs were recorded was associated with the - either actually or potentially - correct vs. incorrect response), Spatial Stimulus-Hand (S-H) Relation in Trial $N$ (i.e., whether the hand from which the MEPs were recorded was ipsilateral vs. contralateral to the stimulus), and Spatial Compatibility in Trial $N-1$.

This analysis revealed significant main effects of type of trial $\left[F(1,17)=15.68, p<.01, \eta_{\mathrm{p}}{ }^{2}=.48\right]$ and $\mathrm{S}-\mathrm{H}$ relation $\left[F(1,17)=24.82, p<.01, \eta_{\mathrm{p}}{ }^{2}=.59\right]$. The interaction between type of trial and hand correctness was significant $[F(1,17)=$ $\left.26.86, p<.01, \eta_{\mathrm{p}}^{2}=.61\right]$ : In the responding trials, the MEPs recorded from the hand associated with the correct, required response were significantly greater than those recorded from the hand associated with the incorrect response $(p<.05)$, whereas there were no significant differences between these two conditions in the nonresponding trials $(p>.05)$. We also found a significant interaction between $N-1$ spatial compatibility and $\mathrm{S}-\mathrm{H}$ relation $\left[F(2,34)=17.60, p<.01, \eta_{\mathrm{p}}{ }^{2}=.50\right]$, which revealed a sequential modulation of the Simon effect in the MEP amplitudes: The difference between the MEPs recorded from the ipsilateral and contralateral hands was greatest after compatible trials, intermediate after neutral trials, and smallest after incompatible trials (Fig. 1). Such an interaction was not modulated by the type of trial $(p>.05)$, which suggests that sequential modulations occurred in both the responding and nonresponding trials.

To explore more in depth the sequential modulations on nonresponding trials, a separate ANOVA was carried out specifically on the MEPs elicited in such trials. Notably, the interaction between the spatial compatibility in trial $N-1$ and the S-H relation in trial $N$ was significant $[F(2,34)=4.13$, $\left.p<.05, \eta_{\mathrm{p}}{ }^{2}=.20\right]$, revealing that, even in the absence of an overt response, the Simon effect was evident in M1 excitability and was modulated by the spatial compatibility of the preceding trial (Fig. 1). More particularly, post-hoc analyses showed that, after compatible trials $N-1$, the Simon effect in M1 excitability was significant, with ipsilateral MEPs being larger than contralateral MEPs $(p<.05)$. This effect was reduced and no longer significant after neutral trials $(p>.05)$. Finally, after incompatible trials, an opposite pattern appeared,

Table 1 Mean values (and standard errors) of reaction times (RTs, in milliseconds) and proportions of correct responses in compatible- and incompatible-responding $(N)$ trials, as a function of the spatial compatibility of the preceding $(N-1)$ trial

\begin{tabular}{|c|c|c|c|c|c|c|}
\hline \multirow[t]{2}{*}{ Dependent Variable } & \multirow[t]{2}{*}{$N-1$ Compatibility } & \multirow{2}{*}{$\begin{array}{l}\text { Compatible } \\
\text { Trials }\end{array}$} & \multirow[t]{2}{*}{ Incompatible Trials } & \multicolumn{3}{|c|}{ ANOVA Factors and Interactions } \\
\hline & & & & $N-1$ Compatibility & $N$ Compatibility & $N-1 \times N$ Compatibility \\
\hline \multirow[t]{3}{*}{ RTs } & Compatible & $400(11)$ & $465(12)$ & \multirow{3}{*}{$\begin{array}{l}F(2,34)=2.31 \\
p>.05 \\
\eta_{\mathrm{p}}^{2}=.12\end{array}$} & \multirow{3}{*}{$\begin{array}{l}F(1,17)=65.88 \\
p<.01 \\
\eta_{\mathrm{p}}{ }^{2}=.79\end{array}$} & \multirow{3}{*}{$\begin{array}{l}F(2,34)=20.74 \\
p<.01 \\
\eta_{\mathrm{p}}{ }^{2}=.54\end{array}$} \\
\hline & Neutral & $419(14)$ & $452(14)$ & & & \\
\hline & Incompatible & $426(14)$ & $431(13)$ & & & \\
\hline \multirow[t]{3}{*}{ Accuracy } & Compatible & $.99(.003)$ & $.93(.012)$ & \multirow{3}{*}{$\begin{array}{l}F(2,34)=2.45 \\
p>.05 \\
\eta_{\mathrm{p}}{ }^{2}=.12\end{array}$} & \multirow{3}{*}{$\begin{array}{l}F(1,17)=25.19 \\
p<.01 \\
\eta_{\mathrm{p}}{ }^{2}=.59\end{array}$} & \multirow{3}{*}{$\begin{array}{l}F(2,34)=6.19 \\
p<.01 \\
\eta_{\mathrm{p}}{ }^{2}=.26\end{array}$} \\
\hline & Neutral & $.98(.004)$ & $.95(.011)$ & & & \\
\hline & Incompatible & $.98(.004)$ & $.97(.007)$ & & & \\
\hline
\end{tabular}

Statistics and associated $p$ values from the ANOVAs of these data are also reported 


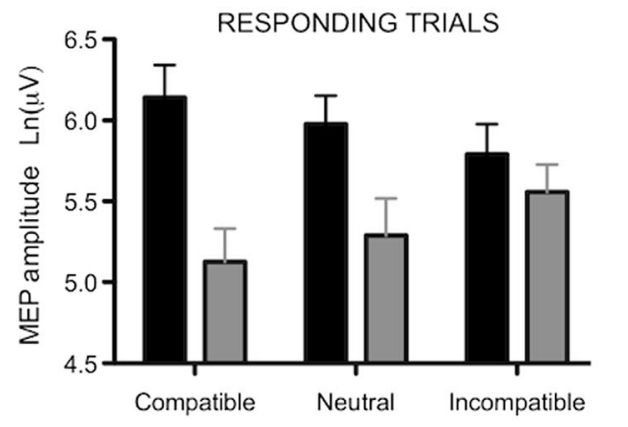

N - 1 condition

Fig. 1 Sequential modulation of the Simon effect on MEP amplitudes. In both the responding and nonresponding trials, the difference between the MEPs recorded in the hands ipsilateral and contralateral to the stimulus

with the ipsilateral MEPs being smaller than contralateral MEPs, even if this difference did not reach statistical significance $(p=.07)$. Interestingly, a test for the linear trend of the ipsilateral versus contralateral MEP differences after compatible, neutral, and incompatible trials revealed that the Simon effect became smaller going from the $N-1$ compatible to incompatible conditions $[F(1,17)=7.26, p<.05]$.

All other main effects and interactions were not statistically significant (all $p \mathrm{~s}>.05$ ), which means that no differences were observed as a function of either the hemisphere stimulated or whether MEPs were observed from the hand associated with the (potentially) correct or incorrect response.

\section{Discussion}

In the present study, we aimed at verifying whether, in conditions under which multiple responses were possible, response conflicts occur and control mechanisms are engaged to solve them, even when there is no intention to execute any response. In other words, what happens when we are involved in a conflict task but, in some trials, we are not required to perform any response?

According to the classical version of the conflict adaptation account, when we intend to perform a given response and there is a conflict between two competing responses, a control mechanism suppresses the direct-route activation of the incorrect response (i.e., the spatially compatible one) in order to accomplish successfully the intended response. The effect of the direct-route suppression can then be observed in the following trial (Akçay \& Hazeltine, 2007, 2011). Accordingly, such a suppression should not occur in nonresponding trials, as they did not imply the intention to select a response (cf. Hommel, Proctor, \& Vu 2004). By contrast, we found that the effect of spatial compatibility on motor cortical excitability was modulated by the compatibility of the previous trial not only for responding pairs, but also for nonresponding pairs.

A sequential modulation of the Simon effect in the MEPs elicited on trials in which participants had to respond to the position was modulated by a sequence effect, which was largest after compatible trials, intermediate after neutral trials, and smallest after incompatible trials

stimulus has been observed in previous studies (Treccani et al., 2016; van Campen et al., 2014). These studies showed that both motor excitatory and inhibitory processes (i.e., ipsilateral response activation and contralateral response inhibition) underlie the Simon effect. In particular, van Campen and collaborators analyzed the temporal changes in $\mathrm{M} 1$ activation and inhibition underlying the resolution of response conflict.

Notably, a sequential modulation of compatibility effects was shown also to occur when participants did not have to emit any response. Indeed, in nonresponding trials after compatible trials, a significant Simon effect on MEPs was observed, indicating that the area of M1 controlling the hand ipsilateral to the stimulus location was more activated than the area controlling the contralateral hand. Thus, even though participants were not required to select any response, the direct route was active, and the mere presentation of lateralized stimuli evoked the activation of the spatially compatible response. Conversely, after incompatible trials, the difference in activation between the areas of M1 controlling, respectively, the hands ipsilateral and contralateral to stimulus position was cancelled. The Simon effect was largest after compatible trials, intermediate (and no longer significant) after neutral trials, and smallest (and even tended to reverse) after incompatible trials.

What do these results suggest? Following the idea of a critical role of the conflict adaptation mechanism in sequential effects, these results suggest that the controlled route, too, was activated on nonresponding trials. Indeed, if the direct route was the only one to act, only the spatially compatible response would have been activated, and no response conflict would have occurred on incompatible trials. Hence, the compatibility of a trial would not have had any consequences on the following trial. However, this was not the case. The sequential modulation of the Simon effect suggests that the two routes were both activated. In incompatible trials, they triggered different responses, thus generating a conflict that led to a reduction of the Simon effect in the following trial.

These results also suggest that the conflict was detected by a control mechanism that, although not necessary, was 
triggered automatically. We can rule out that this mechanism reflects intentional, consciously activated control (cf. Ansorge et al., 2011; Kunde, 2003; Ullsperger, Bylsma, \& Botvinick, 2005), since participants had no intention to select any response (cf. Hommel et al., 2004). Rather, it seems to imply automatic modulation of the activation of the spatially compatible response, based on the compatibility of the previous trial. Our results extend the findings of previous studies (e.g., Desender \& Van Den Bussche, 2012; van Gaal et al., 2010) in revealing that cognitive control can operate not only without awareness of the conflict, but also without an intention to select a response, acting in an automatic fashion.

Before drawing these conclusions, however, we must consider an alternative interpretation of the results. Although the conflict adaptation hypothesis is the most-widely accepted account of sequential effects, these effects have been also explained as the result of episodic memory processes. During the execution of trial $N-1$, the stimulus and response features would be integrated into a common S-R representation, called an event file (Hommel et al., 2004). When the same $N-1$ stimulus features (e.g., a red stimulus on the left) are presented again in trial $N$, the response associated with them in the event file would be reactivated, leading to shorter RTs if this response is the correct one. These complete S-R repetitions (i.e., same stimulus and same response) never occur when the compatibility conditions differ between subsequent trials, whereas they are only possible for $N-1$ compatible $-N$ compatible pairs (which makes compatible trials after other compatible trials even faster) or for $N-1$ incompatible- $N$ incompatible pairs (which speeds up incompatible trials after other incompatible trials), thus increasing and decreasing the Simon effect after compatible and incompatible trials, respectively.

Could this alternative view account for our pattern of data? One might sensibly postulate that the execution of a response is necessary to create an $\mathrm{S}-\mathrm{R}$ event file. If this were the case, this view would hardly explain the sequential modulations observed in the nonresponding trials of our study. However, the feature integration account cannot completely be ruled out, because we cannot exclude that a given response could be linked to a stimulus in an event file, even if such a response were not executed (simply because it was covertly activated). Obviously, we should also conjecture that these (unintentionally created) episodic traces could have an effect on motor cortex activation in the following trial. Future studies might be useful to test and compare these two alternative accounts.

\section{References}

Akçay, C., \& Hazeltine, E. (2007). Conflict monitoring and feature overlap: Two sources of sequential modulations. Psychonomic Bulletin \& Review, 14, 724-748.
Akçay, Ç., \& Hazeltine, E. (2011). Domain-specific conflict adaptation without feature repetitions. Psychonomic Bulletin \& Review, 18 505-511. doi:10.3758/s13423-011-0084-y

Ansorge, U., Fuchs, I., Khalid, S., \& Kunde, W. (2011). No conflict control in the absence of awareness. Psychological Research, 75, 351-365.

Bargh, J. A. (1990). Goal and intent: Goal-directed thought and behavior are often unintentional. Psychological Inquiry, 1, 248-251.

Botvinick, M. M., Braver, T. S., Barch, D. M., Carter, C. S., \& Cohen, J. D. (2001). Conflict monitoring and cognitive control. Psychological Review, 108, 624-652. doi:10.1037/0033-295X.108.3.624

Cheeran, B., Talelli, P., Mori, F., Koch, G., Suppa, A., Edwards, M., \& Rothwell, J. C. (2008). A common polymorphism in the brainderived neurotrophic factor gene (BDNF) modulates human cortical plasticity and the response to rTMS. Journal of Physiology, 586, 5717-5725.

De Gennaro, L., Marzano, C., Veniero, D., Moroni, F., Fratello, F., Curcio, G., \& Rossini, P. M. (2007). Neurophysiological correlates of sleepiness: A combined TMS and EEG study. NeuroImage, 36, $1277-1287$.

Desender, K., \& Van Den Bussche, E. (2012). Is consciousness necessary for conflict adaptation? A state of the art. Frontiers in Human Neuroscience, 6, 3. doi:10.3389/fnhum.2012.00003

Egner, T., \& Hirsch, J. (2005). Cognitive control mechanisms resolve conflict through cortical amplification of task-relevant information. Nature Neuroscience, 8, 1784-1790. doi:10.1038/nn1594

Eimer, M., \& Schlaghecken, F. (1998). Effects of masked stimuli on motor activation: Behavioral and electrophysiological evidence. Journal of Experimental Psychology: Human Perception and Performance, 24, 1737-1747. doi:10.1037/0096-1523.24.6. 1737

Hommel, B., Proctor, R. W., \& Vu, K.-P. L. (2004). A feature-integration account of sequential effects in the Simon task. Psychological Research, 68, 1-17. doi:10.1007/s00426-003-0132-y

Iyer, M. B., Schleper, N., \& Wassermann, E. M. (2003). Priming stimulation enhances the depressant effect of low-frequency repetitive transcranial magnetic stimulation. Journal of Neuroscience, 23, $10867-10872$.

Kinoshita, S., Mozer, M. C., \& Forster, K. I. (2011). Dynamic adaptation to history of trial difficulty explains the effect of congruency proportion on masked priming. Journal of Experimental Psychology: General, 140, 622-636. doi:10.1037/a0024230

Klein, P.-A., Petitjean, C., Olivier, E., \& Duque, J. (2014). Top-down suppression of incompatible motor activations during response selection under conflict. Neurolmage, 86, 138-149.

Kunde, W. (2003). Sequential modulations of stimulus-response correspondence effects depend on awareness of response conflict. Psychonomic Bulletin \& Review, 10, 198-205. doi:10.3758/ BF03196485

Lau, H. C., \& Passingham, R. E. (2007). Unconscious activation of the cognitive control system in the human prefrontal cortex. Journal of Neuroscience, 27, 5805-5811.

Lu, C.-H., \& Proctor, R. W. (1995). The influence of irrelevant location information on performance: A review of the Simon and spatial Stroop effects. Psychonomic Bulletin \& Review, 2, 174-207. doi: 10.3758/BF03210959

Mayr, U. (2004). Conflict, consciousness, and control. Trends in Cognitive Sciences, 8, 145-148.

Michelet, T., Duncan, G. H., \& Cisek, P. (2010). Response competition in the primary motor cortex: Corticospinal excitability reflects response replacement during simple decisions. Journal of Neurophysiology, 104, 119-127.

Nielsen, J. F. (1996). Logarithmic distribution of amplitudes of compound muscle action potentials evoked by transcranial magnetic stimulation. Journal of Clinical Neurophysiology, 13, 423-434. 
Oldfield, R. C. (1971). The assessment and analysis of handedness: The Edinburgh inventory. Neuropsychologia, 9, 97-113. doi:10.1016/ 0028-3932(71)90067-4

Ridderinkhof, R. (2002). Activation and suppression in conflict tasks: Empirical clarification through distributional analyses. In W. Prinz \& B. Hommel (Eds.), Attention and performance XIX: Common mechanisms in perception and action (pp. 494-519). Oxford: Oxford University Press.

Rossini, P. M., Barker, A. T., Berardelli, A., Caramia, M. D., Caruso, G., Cracco, R. Q., \& Tomberg, C. (1994). Non-invasive electrical and magnetic stimulation of the brain, spinal cord and roots: Basic principles and procedures for routine clinical application. Report of an IFCN committee. Electroencephalography and Clinical Neurophysiology, 91, 79-92. doi:10.1016/0013-4694(94)90029-9

Stürmer, B., Siggelkow, S., Dengler, R., \& Leuthold, H. (2000). Response priming in the Simon paradigm: A transcranial magnetic stimulation study. Experimental Brain Research, 135, 353-359.

Ticini, L. F., Schütz-Bosbach, S., Weiss, C., Casile, A., \& Waszak, F. (2012). When sounds become actions: Higher-order representation of newly learned action sounds in the human motor system. Journal of Cognitive Neuroscience, 24, 464-474.

Treccani, B., Cona, G., Milanese, N., \& Umiltà, C. A. (2016). Sequential modulation of response activation and inhibition in a response conflict task: A single-pulse transcranial magnetic stimulation (TMS) study. Manuscript submitted for publication.

Ullsperger, M., Bylsma, L. M., \& Botvinick, M. M. (2005). The conflict adaptation effect: It's not just priming. Cognitive, Affective, \& Behavioral Neuroscience, 5, 467-472. doi:10.3758/CABN.5.4.467

Valle-Inclán, F. (1996). The Simon effect and its reversal studied with event-related potentials. International Journal of Psychophysiology, $23,41-53$.

van Campen, A. D., Keuken, M. C., van den Wildenberg, W. P., \& Ridderinkhof, K. R. (2014). TMS over M1 reveals expression and selective suppression of conflicting action impulses. Journal of Cognitive Neuroscience, 26, 1-15. doi:10.1162/jocn_a_00482

van Gaal, S., Lamme, V. A. F., \& Ridderinkhof, $\overline{\text { K}}$. R. (2010). Unconsciously triggered conflict adaptation. PLoS ONE, 5, e11508. doi:10.1371/journal.pone.0011508

Wassermann, E. M. (1998). Risk and safety of repetitive transcranial magnetic stimulation: Report and suggested guidelines from the International Workshop on the Safety of Repetitive Transcranial Magnetic Stimulation. Electroencephalography and Clinical Neurophysiology, 108, 1-16.

Wassermann, E. M. (2002). Variation in the response to transcranial magnetic brain stimulation in the general population. Clinical Neurophysiology, 113, 1165-1171. 